\title{
Some Noncommutative Matrix Algebras Arising in the Bispectral Problem
}

\author{
F. Alberto GRÜNBAUM
}

Department of Mathematics, University of California, Berkeley, CA 94720 USA

E-mail: grunbaum@math.berkeley.edu

URL: http://math. berkeley.edu/ grunbaum/

Received May 01, 2014, in final form July 17, 2014; Published online July 24, 2014

http://dx.doi.org/10.3842/SIGMA.2014.078

\begin{abstract}
I revisit the so called "bispectral problem" introduced in a joint paper with Hans Duistermaat a long time ago, allowing now for the differential operators to have matrix coefficients and for the eigenfunctions, and one of the eigenvalues, to be matrix valued too. In the last example we go beyond this and allow both eigenvalues to be matrix valued.
\end{abstract}

Key words: noncommutative algebras; bispectral problem

2010 Mathematics Subject Classification: 13N10; 16S32; 35P05

\section{Introduction}

The characterization of certain commutative algebras of differential operators goes back to pioneering work of Schur and Burchnall jointly with Chaundy in the early part of the twenty century, see $[3,4,5,32]$. The subject remained dormant until the 1970's where the study of the Kortewegde Vries equation revived and enriched the interaction between algebraic geometry, differential operators, group representation theory and many other parts of mathematics. Suddenly the KdV equation, and related ones, such as the Toda and KP equations, started showing up in the solution of many "unrelated" problems. For an account of this revival see, for instance, [24, 27, 28].

One such problem was posed and solved in [7] and has become known as the "bispectral problem".

It consists of finding all scalar coefficient ordinary differential operators $L$ and $B$ in the variables $x$ and $z$ respectively such that there is a common eigenfunction $\psi(x, z)$ satisfying the eigenvalue equations

$$
L \psi=p(z) \psi, \quad B \psi=\theta(x) \psi
$$

for nonconstant scalar valued functions $p$ and $\theta$.

More explicitly we wanted to find all nontrivial instances where a function $\psi(x, z)$ satisfies

$$
L\left(x, \frac{d}{d x}\right) \psi(x, z) \equiv\left(-\left(\frac{d}{d x}\right)^{2}+V(x)\right) \psi(x, z)=z \psi(x, z)
$$

as well as

$$
B\left(z, \frac{d}{d z}\right) \psi(x, z) \equiv\left(\sum_{i=0}^{M} b_{i}(z)\left(\frac{d}{d z}\right)^{i}\right) \psi(x, z)=\theta(x) \psi(x, z) .
$$

All the scalar valued functions $V(x), b_{i}(z), \theta(x)$ are, in principle, arbitrary except for smoothness assumptions. Notice that here $M$ is arbitrary (finite). The operator $L$ could be of higher order, but in [7] we stick to order two. 
The paper with Duistermaat starts by proving the importance of the so called "ad-conditions", see (2), an extremely complicated system of non-linear ordinary differential equations in the coefficients of $L$ and the function $\theta$. This line of attack, which produced the first few examples of bispectral situations, would have led nowhere if it were not for the totally unexpected observation that the (rational solutions of the) KdV equation was lurking around.

Maybe the most remarkable fact is that the answer to this innocent looking question hides connections with many important developments in the area of integrable systems. Among these, one finds a useful role for the Darboux process, the notion of monodromy and finally the appearance of certain flows, such as KP, that play a central role. The set of all possible $L$ and the algebra of all differential operators $B$ going with a given $L$ was explicitly characterized in [7] in terms of an appropriate "tau" function. In the case discussed in [7] there are two families of potentials $V(x)$ going with bundles of rank one and two respectively. In the first case the "tau" functions go back to Schur and one is dealing with the KdV flows; in the second case, called the even family in [7], one is dealing with the Virasoro flows (the master symmetries of $\mathrm{KdV}$ ).

The "tau" functions were given explicitly in [7] and the observation regarding the second flow came later, see [43]. As far as I know no one has yet traced these second "tau" functions to something like characters of certain group representation.

As we just mentioned, in the scalar valued case the commutative algebra of all differential operators $B$ going with a fixed $L$ is explicitly characterized in terms of an appropriate "tau" function. This description of this commutative algebra is given below. Notice that we also have a characterization of the possible potentials $V(x)$ that appear in the operator $L$. Nothing like this is available in the noncommutative case, all that we have at this point are a few examples, as we will see later.

For the $V(x)$ in the KdV family we have

$$
V(x)=\sum_{p \in \mathcal{P}} \frac{\nu_{p}\left(\nu_{p}+1\right)}{(x-p)^{2}}
$$

with $\mathcal{P}$ a finite subset of $\mathbb{C}$, and $\nu_{p} \in \mathbb{Z}_{>0}$ for $p \in \mathcal{P}$ being such that

$$
\sum_{\substack{q \in \mathcal{P} \\ q \neq p}} \frac{\nu_{q}\left(\nu_{q}+1\right)}{(q-p)^{2 j+1}}=0 \quad \text { for } 1 \leq j \leq \nu_{p} \quad \text { and each } \quad p \in \mathcal{P} .
$$

One can also write

$$
V(x)=-2\left(\frac{\theta^{\prime}(x)}{\theta(x)}\right)^{\prime}
$$

Here

$$
\theta(x)=\prod_{p}(x-p)^{\frac{1}{2} \nu_{p}\left(\nu_{p}+1\right)}
$$

and $p$ runs over $\mathcal{P}$.

For these potentials we have the following characterization of the algebra of differential operators in the spectral variable:

The eigenfunction $\psi(x, z)$ satisfies an equation of the form $B\left(z, \partial_{z}\right) \psi(x, z)=\theta(x) \psi(x, z)$ if and only if the polynomial $\theta$ has the property that

$$
\theta^{(2 j-1)}(p)=0 \quad \text { for all } 1 \leq j \leq \nu_{p}, \quad \text { for each pole } p \in \mathbb{C} \text { of } V .
$$

For the $V(x)$ in the even family we have a similar situation. 
In [16] ones sees how a q-version (replacing differential operators by $q$-differences) of the scalar valued bispectral problem touches on some very important work of Dick Askey. This is one more instance of the wide impact of "special functions" in several parts of mathematics.

Several authors have made important contributions to illuminate this "bispectral problem" from different perspectives. Since this paper is not intended to be a survey I have restrained myself from mentioning most of this very nice work. Apologies, apologies, .... Instead of surveying all that is known in the scalar case I want to take a jump back in time to the early 1980 's and pose some conjectures dealing with a matrix valued version of the problem that Hans Duistermaat and I started looking at back then. In this much more difficult situation there is still no clear picture of a complete solution.

As pointed out in [1] the first work on a matrix valued version of the bispectral problem is due to J.P. Zubelli (in his Berkeley Ph.D. thesis), see for instance [42]. For more recent work along these lines, see $[9,31]$.

The transition to a matrix valued version of the same problem, with one of the two variables becoming discrete, is featured for instance in a series of papers with Pacharoni and Tirao, see [20, 21], involving matrix valued spherical functions for certain symmetric spaces. Here the spherical functions (properly "packaged") give rise to matrix valued orthogonal polynomials (a notion due to M.G. Krein $[25,26]$ ) that happen to satisfy difference as well as differential equations. Other examples of matrix valued orthogonal polynomials were given in joint work with Duran [8]. In all these papers, starting with [20] the observation is made that it is important that the operators $\mathcal{L}$ and $\mathcal{B}$ should act on the left and the right respectively. There is no other way to insure the commutativity of these two operators with matrix valued coefficients. This is also noticed in a short paper with Iliev [17], where we try to use the ad-conditions.

The problem is now formulated in the form

$$
\mathcal{L} \Psi=p(z) \Psi, \quad \Psi \mathcal{B}=\Theta(x) \Psi
$$

for nonconstant functions $p$ and $\Theta$ which are scalar and matrix valued respectively. The differential operators have matrix valued coefficients and they act on the matrix valued eigenfunction $\Psi$.

The problem of classifying the noncommutative algebra of differential operators $\mathcal{B}$ going with a fixed $\mathcal{L}$, i.e., with a fixed family of matrix valued orthogonal polynomials, was first considered in a joint paper with Castro [6]. There we formulate conjectures about the structure of the algebra in a few basic examples. For a matrix valued version of the Hermite polynomials the conjecture was eventually proved by Tirao [37] in a paper that gives a complete "nice presentation" of this algebra in terms of generators and relations.

For a fixed $\mathcal{L}$ (going with a fixed family of matrix valued orthogonal polynomials) the algebra in question is isomorphic to the algebra of all matrix valued polynomials $\Theta(x)$ satisfying the "ad-conditions" mentioned in the introduction

$$
(\operatorname{ad} \mathcal{L})^{m+1}(\Theta(x))=0
$$

for some $m$. This is the starting point of my work with Duistermaat and has been seen to hold in the matrix case too, see [23].

My original motivation for the bispectral problem came from a very concrete application: the remarkable observation by D. Slepian, H. Landau and H. Pollak at Bell Labs back in the 1960's that the integral "time-and-band limiting" operator of Claude Shannon allowed for an explicit commuting differential operator, see [13, 15, 35, 36].

In a few cases I have been able to go back from bispectral situations to the motivating problem, see $[11,12,14,19]$, see also $[29,30]$. Very recently this has been accomplished for the first time in a noncommutative set-up, see [22].

The work in [19] has been picked up in the applied literature, see for instance [33, 34]. 


\section{Contents of the paper}

To the best of my knowledge the first papers to consider the case of two differential operators acting from different directions are [2] and [1]. These papers acknowledge that this idea had been advanced in the papers mentioned above dealing with differential and difference operators.

The first paper [2] includes two examples: in each case $\mathcal{L}$ has order two and $\mathcal{B}$ has order three in one case and four in the other one. Here the authors show the bispectrality of two examples given in [10] of (matrix) Schrödinger operators with trivial monodromy. Note that in the abstract of [2] both eigenvalues are allowed to be matrix valued. In the actual examples in [2] they are both scalar valued and in the body of the paper (see the proof of Theorem 3.6) the right hand side of the equation involving $\mathcal{B}$ is written differently that the way it appears in the abstract. In a recent conversation with J. Liberati he seems to prefer the second version. I prefer the other one, the one in their abstract.

The second paper [1] exhibits an example arising from spin Calogero systems and the use of the Wilson bispectral involution, a remarkably useful tool, see [38, 39, 40, 41]. In [1] the operator $\mathcal{L}$ has order four and $\mathcal{B}$ has order six. The problem is formulated there with both eigenvalues taken as scalar valued functions. Notice that in some of the examples of matrix bispectrality that arise in $[6,8,17,20,21]$ as well as others in the context of matrix valued orthogonal polynomials this is not necessarily the case, and allowing (at least) one of the eigenvalues to be matrix valued is the natural thing to do.

I first deal with the first example in [2], see also [10], where a bispectral situation is given involving a matrix valued eigenfunction but restricted to the case of scalar valued eigenvalues. I do allow for arbitrary matrix valued polynomial $\Theta(x)$.

I then consider an analog of this two-by-two example in a three-by-three step-up. In each case I give an explicit conjecture about the algebra of all possible $\Theta(x)$, or equivalently all possible operators $\mathcal{B}$.

Finally I give an algebra and a conjecture about all possible matrix valued eigenvalues related to an example which is connected to spin Calogero models but more elaborate than the one in [1].

\section{The first example}

Take for $\Psi(x, z)$ the matrix valued function

$$
\Psi(x, z)=e^{x z}\left(\begin{array}{cc}
z-x^{-1} & x^{-2} \\
0 & z-x^{-1}
\end{array}\right)
$$

and consider all instances of matrix valued polynomials $\Theta(x)$ and differential operators $\mathcal{B}$ (with matrix coefficients $\left.b_{i}(z)\right)$ such that

$$
\Psi \mathcal{B} \equiv \sum_{i=1}^{m}\left(\partial_{z}^{i} \Psi\right) b_{i}=\Theta(x) \Psi(x) .
$$

In this case one has

$$
\mathcal{L} \Psi=-z^{2} \Psi
$$

with

$$
\mathcal{L}=-\partial_{x}^{2}+2\left(\begin{array}{cc}
x^{-2} & -2 x^{-3} \\
0 & x^{-2}
\end{array}\right)
$$

In other words for this specific differential operator in the variable $x$ we are asking for all bispectral "partners" of $\mathcal{L}$. 
In [2] one finds that one such pair $(\mathcal{B}, \Theta)$ is given by

$$
\mathcal{B}=\partial_{z}^{3}-3 \partial_{z}^{2} \frac{1}{z}+3 \partial_{z} \frac{1}{z^{2}}+3\left(\begin{array}{cc}
0 & z^{-2} \\
0 & 0
\end{array}\right)
$$

and $\Theta(x)$ the scalar-valued polynomial

$$
\Theta(x)=x^{3} .
$$

For operators $\mathcal{L}$ of the form

$$
\mathcal{L}=-\partial_{x}^{2}+\mathbb{U}(x)
$$

with a matrix valued potential $\mathbb{U}(x)$ one can argue as in [7] (using the ad-conditions) and conclude that $\Theta(x)$ has to be a polynomial with matrix valued coefficients. The "matrix valued" eigenvalue $\Theta(x)$ has to satisfy lots of other restrictions for the equation

$$
\Psi \mathcal{B}=\Theta(x) \Psi
$$

to hold, and the problem at hand is to describe explicitly all these $\Theta(x)$. An important observation is that this set of $\Theta$ 's form a noncommutative algebra of polynomials in $x$ and the algebra of the corresponding differential operators $\mathcal{B}$ is isomorphic to it.

Conjecture 1. In our case the set of all $\Theta$ 's is the algebra of all polynomials of the form

$$
\left(\begin{array}{cc}
r_{0}^{11} & r_{0}^{12} \\
0 & r_{0}^{11}
\end{array}\right)+\left(\begin{array}{cc}
r_{1}^{11} & r_{1}^{12} \\
0 & r_{1}^{11}
\end{array}\right) x+\left(\begin{array}{cc}
r_{2}^{11} & r_{2}^{12} \\
r_{1}^{11} & r_{2}^{22}
\end{array}\right) x^{2}+\left(\begin{array}{cc}
r_{3}^{11} & r_{3}^{12} \\
r_{2}^{22}+r_{2}^{11}-r_{1}^{12} & r_{3}^{22}
\end{array}\right) x^{3}+x^{4} P(x),
$$

where $P(x)$ is an arbitrary $2 \times 2$ matrix valued polynomial and all the variables $r_{0}^{11}, r_{0}^{12}, r_{1}^{11}$, $r_{1}^{12}, r_{2}^{11}, r_{2}^{12}, r_{2}^{22}, r_{3}^{11}, r_{3}^{12}, r_{3}^{22}$ are arbitrary.

Moreover, for each such $\Theta$ one can give an explicit expression for the corresponding operator $\mathcal{B}$.

Comments. It is not hard to check that the set of polynomials $\Theta(x)$ given above forms an algebra. It is also not hard to propose a list of generators for the algebra and certain relations among them. The remaining open problem is to prove the conjecture and to give a "nice description" of the algebra in terms of generators and relations.

The situation is analogous to what was put forth in [6], namely a conjecture about the algebra and a collection of generators for a few examples. The proof of these conjectures and a "nice description" of the algebra was only done, for one of the examples put forward in [6] and [37].

\section{Second example}

Take for $\Psi(x, z)$ the matrix valued function

$$
\Psi(x, z)=\left[\partial_{x}-\left(\begin{array}{ccc}
x^{-1} & -x^{-2} & x^{-3} \\
0 & x^{-1} & -x^{-2} \\
0 & 0 & x^{-1}
\end{array}\right)\right] e^{x z} I=e^{x z}\left(\begin{array}{ccc}
z-x^{-1} & x^{-2} & -x^{-3} \\
0 & z-x^{-1} & x^{-2} \\
0 & 0 & z-x^{-1}
\end{array}\right) .
$$

Here one can see that

$$
\mathcal{L} \Psi=-z^{2} \Psi
$$

with

$$
\mathcal{L}=-\partial_{x}^{2}+2\left(\begin{array}{ccc}
x^{-2} & -2 x^{-3} & 3 x^{-4} \\
0 & x^{-2} & -2 x^{-3} \\
0 & 0 & x^{-2}
\end{array}\right)
$$


Conjecture 2. The algebra of all matrix valued polynomials $\Theta(x)$ for which there exist some operator $\mathcal{B}$ with

$$
\Psi \mathcal{B}=\Theta(x) \Psi
$$

is the algebra of all polynomials of the form

$$
\begin{aligned}
& \left(\begin{array}{ccc}
r_{0}^{11} & r_{0}^{12} & r_{0}^{13} \\
0 & r_{0}^{22} & r_{0}^{23} \\
0 & 0 & r_{0}^{11}
\end{array}\right)+\left(\begin{array}{ccc}
r_{1}^{11} & r_{1}^{12} & r_{1}^{13} \\
r_{0}^{22}-r_{0}^{11} & r_{1}^{22} & r_{1}^{23} \\
0 & r_{0}^{22}-r_{0}^{11} & r_{1}^{11}+r_{0}^{23}-r_{0}^{12}
\end{array}\right) x \\
& +\left(\begin{array}{ccc}
r_{2}^{11} & r_{2}^{12} & r_{2}^{13} \\
r_{1}^{22}-r_{1}^{11}-r_{0}^{23}+r_{0}^{12} & r_{2}^{22} & r_{2}^{23} \\
r_{0}^{22}-r_{0}^{11} & r_{1}^{22}-r_{1}^{11} & r_{2}^{11}+r_{1}^{23}-r_{1}^{12}
\end{array}\right) x^{2} \\
& +\left(\begin{array}{ccc}
r_{3}^{11} & r_{3}^{12} & r_{3}^{13} \\
r_{3}^{21} & r_{3}^{22} & r_{3}^{23} \\
r_{1}^{22}-2 r_{1}^{11}-r_{0}^{23}+r_{0}^{12} & r_{3}^{32} & r_{3}^{33}
\end{array}\right) x^{3} \\
& +\left(\begin{array}{ccc}
r_{4}^{11} & r_{4}^{12} & r_{4}^{13} \\
r_{4}^{21} & r_{4}^{22} & r_{4}^{23} \\
r_{3}^{32}+r_{3}^{21}-r_{2}^{22}-r_{2}^{11}+r_{1}^{12} & r_{4}^{32} & r_{4}^{33}
\end{array}\right) x^{4} \\
& +\left(\begin{array}{ccc}
r_{5}^{11} & r_{5}^{12} & r_{5}^{13} \\
r_{5}^{21} & r_{5}^{22} & r_{5}^{23} \\
r_{4}^{32}+r_{4}^{21}-r_{3}^{33}-r_{3}^{22}-r_{3}^{11}+r_{2}^{23}+r_{2}^{12}-r_{1}^{13} & r_{5}^{32} & r_{5}^{33}
\end{array}\right) x^{5}+x^{6} P(x),
\end{aligned}
$$

where $P(x)$ is an arbitrary $3 \times 3$ matrix valued polynomial and all the variables $r_{0}^{11}, r_{0}^{12}, \ldots, r_{5}^{33}$ are arbitrary.

Once again one can prove by an explicit computation that this set of $\Theta$ 's forms an algebra and one can single out generators and some relations among them. What is needed is a proof of the conjecture and a "nice presentation" in terms of generation and relations. Here is a very simple case of the general result above. We can check that

$$
\frac{\partial^{2} \Psi}{\partial z^{2}}\left(\begin{array}{ccc}
0 & 0 & 0 \\
0 & 0 & 0 \\
1 & 0 & 0
\end{array}\right)+\frac{\partial \Psi}{\partial z}\left(\begin{array}{ccc}
0 & 0 & 0 \\
1 & 0 & 0 \\
-2 z^{-1} & 1 & 0
\end{array}\right)+\Psi\left(\begin{array}{ccc}
1 & 0 & 0 \\
-2 z^{-1} & 2 & 0 \\
0 & 0 & 1
\end{array}\right)=\left(\begin{array}{ccc}
1 & 0 & 0 \\
x & 2 & 0 \\
x^{2} & x & 1
\end{array}\right) \Psi
$$

\section{Third example}

We consider now a situation that will be explained fully in [18]. It comes about by looking at examples linked to the spin Calogero systems discussed for instance in [1].

Consider the function $\Psi(x, z)$ given by

$$
\Psi(x, z)=\frac{e^{x z}}{(x-2) x z}\left(\begin{array}{cc}
\frac{x^{3} z^{2}-2 x^{2} z^{2}-2 x^{2} z+3 x z+2 x-2}{x z} & \frac{1}{x} \\
\frac{(x z-2)}{z} & x^{2} z-2 x z-x+1
\end{array}\right),
$$

which satisfies

$$
\mathcal{L} \Psi=\Psi F(z)
$$


with

$$
\mathcal{L} \equiv\left(\begin{array}{ll}
0 & 0 \\
0 & 1
\end{array}\right) \partial_{x}^{2}+\left(\begin{array}{cc}
0 & \frac{1}{(x-2) x^{2}} \\
-\frac{1}{x-2} & 0
\end{array}\right) \partial_{x}+\left(\begin{array}{cc}
-\frac{1}{x^{2}(x-2)^{2}} & \frac{x-1}{x^{3}(x-2)^{2}} \\
\frac{2 x-1}{x(x-2)^{2}} & -\frac{2 x^{2}-4 x+3}{x^{2}(x-2)^{2}}
\end{array}\right)
$$

and

$$
F(z)=\left(\begin{array}{cc}
0 & 0 \\
0 & z^{2}
\end{array}\right)
$$

We can now check that

$$
\frac{\partial^{2} \Psi}{\partial z^{2}}\left(\begin{array}{cc}
0 & 0 \\
-\frac{2 z+1}{z} & 0
\end{array}\right)+\frac{\partial \Psi}{\partial z}\left(\begin{array}{cc}
1 & 0 \\
\frac{2(z-1)}{z^{2}} & 1
\end{array}\right)+\Psi\left(\begin{array}{cc}
-z^{-1} & 0 \\
6 z^{-3} & z^{-1}
\end{array}\right)=\left(\begin{array}{cc}
x & 0 \\
x^{2}(x-2) & x
\end{array}\right) \Psi .
$$

We are thus dealing with a situation of the following kind

$$
\mathcal{L} \Psi=\Psi F(z), \quad \Psi \mathcal{B}=\Theta(x) \Psi
$$

for nonconstant matrix valued functions $F$ and $\Theta$. As in (1) the differential operators have matrix valued coefficients and they act on the matrix valued eigenfunction $\Psi$.

One can see that, for the $\Psi(x, z)$ above, the algebra of all $F(z)$ such that for some $\mathcal{L}$ one has

$$
\mathcal{L} \Psi=\Psi F(z)
$$

is given by polynomials in $z$ of the form

$$
\left(\begin{array}{cc}
a & 0 \\
b-a & b
\end{array}\right)+\left(\begin{array}{cc}
c & c \\
a--b-c & -c
\end{array}\right) z+\left(\begin{array}{cc}
a-b-c & c+a-b \\
d & e
\end{array}\right)\left(z^{2}\right) / 2+z^{3} P(z),
$$

where $P(z)$ is an arbitrary $2 \times 2$ matrix valued polynomial and all the variables $a, b, c, d, e$ are arbitrary.

It is not hard to see that this forms an algebra for which, once again, a nice description in terms of generators and relations remains a challenge.

\section{Acknowledgements}

The author is extremely grateful to referees who did an outstanding job in suggesting improvements to an earlier version of this paper.

\section{References}

[1] Bergvelt M., Gekhtman M., Kasman A., Spin Calogero particles and bispectral solutions of the matrix KP hierarchy, Math. Phys. Anal. Geom. 12 (2009), 181-200, arXiv:0806.2613.

[2] Boyallian C., Liberati J.I., Matrix-valued bispectral operators and quasideterminants, J. Phys. A: Math. Theor. 41 (2008), 365209, 11 pages.

[3] Burchnall J.L., Chaundy T.W., Commutative ordinary differential operators, Proc. London Math. Soc. 21 (1923), 420-440.

[4] Burchnall J.L., Chaundy T.W., Commutative ordinary differential operators, Proc. R. Soc. Lond. Ser. A 118 (1928), 557-583.

[5] Burchnall J.L., Chaundy T.W., Commutative ordinary differential operators. II. The identity $P^{n}=Q^{m}$, Proc. R. Soc. Lond. Ser. A 134 (1931), 471-485. 
[6] Castro M.M., Grünbaum F.A., The algebra of differential operators associated to a family of matrixvalued orthogonal polynomials: five instructive examples, Int. Math. Res. Not. 2006 (2006), Art. ID 47602, 33 pages.

[7] Duistermaat J.J., Grünbaum F.A., Differential equations in the spectral parameter, Comm. Math. Phys. 103 (1986), 177-240.

[8] Durán A.J., Grünbaum F.A., Orthogonal matrix polynomials satisfying second-order differential equations, Int. Math. Res. Not. 2004 (2004), no. 10, 461-484.

[9] Gohberg I., Kaashoek M.A., Sakhnovich A.L., Canonical systems with rational spectral densities: explicit formulas and applications, Math. Nachr. 194 (1998), 93-125.

[10] Goncharenko V.M., Veselov A.P., Monodromy of the matrix Schrödinger equations and Darboux transformations, J. Phys. A: Math. Gen. 31 (1998), 5315-5326.

[11] Grünbaum F.A., A new property of reproducing kernels for classical orthogonal polynomials, J. Math. Anal. Appl. 95 (1983), 491-500.

[12] Grünbaum F.A., Some new explorations into the mystery of time and band limiting, Adv. in Appl. Math. 13 (1992), 328-349.

[13] Grünbaum F.A., Time-band limiting and the bispectral problem, Comm. Pure Appl. Math. 47 (1994), 307-328.

[14] Grünbaum F.A., Band-time-band limiting integral operators and commuting differential operators, St. Petersburg Math. J. 8 (1997), 93-96.

[15] Grünbaum F.A., Some bispectral musings, in The Bispectral Problem (Montreal, PQ, 1997), CRM Proc. Lecture Notes, Vol. 14, Amer. Math. Soc., Providence, RI, 1998, 31-45.

[16] Grünbaum F.A., Haine L., Some functions that generalize the Askey-Wilson polynomials, Comm. Math. Phys. 184 (1997), 173-202.

[17] Grünbaum F.A., Iliev P., A noncommutative version of the bispectral problem, J. Comput. Appl. Math. 161 (2003), 99-118.

[18] Grünbaum F.A., Jones V.F.R., Zubelli J., On the bimodule structure of the bispectral problem, in preparation.

[19] Grünbaum F.A., Longhi L., Perlstadt M., Differential operators commuting with finite convolution integral operators: some nonabelian examples, SIAM J. Appl. Math. 42 (1982), 941-955.

[20] Grünbaum F.A., Pacharoni I., Tirao J., Matrix valued spherical functions associated to the complex projective plane, J. Funct. Anal. 188 (2002), 350-441, math.RT/0108042.

[21] Grünbaum F.A., Pacharoni I., Tirao J., Matrix valued orthogonal polynomials of the Jacobi type, Indag. Math. (N.S.) 14 (2003), 353-366.

[22] Grünbaum F.A., Pacharoni I., Zurrian I., Time and band limiting in a noncommutative context, in preparation.

[23] Grünbaum F.A., Tirao J., The algebra of differential operators associated to a weight matrix, Integral Equations Operator Theory 58 (2007), 449-475.

[24] Harnad J., Kasman A. (Editors), The bispectral problem, CRM Proceedings 8 Lecture Notes, Vol. 14, Amer. Math. Soc., Providence, RI, 1998.

[25] Krein M., Infinite $J$-matrices and a matrix-moment problem, Doklady Akad. Nauk SSSR 69 (1949), 125-128.

[26] Krein M.G., Fundamental aspects of the representation theory of Hermitian operators with deficiency index (m,m), Amer. Math. Soc. Translations Ser. II, Vol. 97, Amer. Math. Soc., Providence, RI, 1971, $75-143$.

[27] Krichever I.M., Algebraic curves and nonlinear difference equations, Russ. Math. Surv. 33 (1978), no. 4, 255-256.

[28] Mumford D., An algebro-geometric construction of commuting operators and of solutions to the Toda lattice equation, Korteweg-de Vries equation and related nonlinear equation, in Proceedings of the International Symposium on Algebraic Geometry (Kyoto Univ., Kyoto, 1977), Kinokuniya Book Store, Tokyo, 1978, $115-153$.

[29] Perlstadt M., Chopped orthogonal polynomial expansions - some discrete cases, SIAM J. Algebraic Discrete Methods 4 (1983), 94-100.

[30] Perlstadt M., A property of orthogonal polynomial families with polynomial duals, SIAM J. Math. Anal. 15 (1984), 1043-1054. 
[31] Sakhnovich A., Zubelli J.P., Bundle bispectrality for matrix differential equations, Integral Equations Operator Theory 41 (2001), 472-496.

[32] Schur I., Über vertauschbare lineare Differentialausdrücke, Sitzungsber. Berl. Math. Ges. 4 (1905), 2-8.

[33] Simons F.J., Dahlen F.A., Spherical Slepian functions on the polar gap in geodesy, Geophys. J. Int. 166 (2006), 1039-1061, math.ST/0603271.

[34] Simons F.J., Dahlen F.A., Wieczorek M.A., Spatiospectral concentration on a sphere, SIAM Rev. 48 (2006), 504-536, math.CA/0408424.

[35] Slepian D., On bandwidth, Proc. IEEE 64 (1976), 292-300.

[36] Slepian D., Some comments on Fourier analysis, uncertainty and modeling, SIAM Rev. 25 (1983), 379-393.

[37] Tirao J., The algebra of differential operators associated to a weight matrix: a first example, in Groups, Algebras and Applications, Contemp. Math., Vol. 537, Amer. Math. Soc., Providence, RI, 2011, $291-324$.

[38] Wilson G., Algebraic curves and soliton equations, in Geometry Today (Rome, 1984), Progr. Math., Vol. 60, Birkhäuser Boston, Boston, MA, 1985, 303-329.

[39] Wilson G., Bispectral commutative ordinary differential operators, J. Reine Angew. Math. 442 (1993), 177-204.

[40] Wilson G., Collisions of Calogero-Moser particles and an adelic Grassmannian, Invent. Math. 133 (1998), $1-41$.

[41] Wilson G., The complex Calogero-Moser and KP systems, in Calogero-Moser-Sutherland Models (Montréal, QC, 1997), CRM Ser. Math. Phys., Springer, New York, 2000, 539-548.

[42] Zubelli J.P., Differential equations in the spectral parameter for matrix differential operators, Phys. D 43 (1990), 269-287.

[43] Zubelli J.P., Magri F., Differential equations in the spectral parameter, Darboux transformations and a hierarchy of master symmetries for KdV, Comm. Math. Phys. 141 (1991), 329-351. 